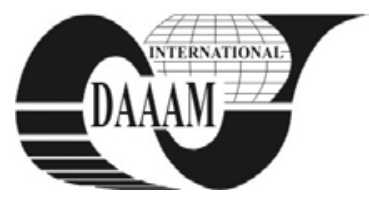

\title{
APPROACHES FOR SOLVING PRODUCTION PLANNING AND SCHEDULING PROBLEMS USING GENETIC ALGORITHMS
}

\author{
CIROVIC, I[van]; SIMEONOV, S[imeon]; STANO, P[avol] \& PFAFF, O[ndrej]
}

\begin{abstract}
Manufacturers are today faced with increasing market competition. Acknowledging considerable pressure by competition, efficient planning and scheduling of production therefore become essential requirements. Satisfying customers' needs and, moreover, benefitting economically, is thus owed to detailed plans of rational production, sales and distribution. Understanding planning, allocating resources and controlling processes of production are indispensable for success in business activities. In conditions of fast changes, when increasingly less time is available for optimal organization of production, it is a must to implement methods (algorithms) utilized towards finding an appropriate solution with relatively low number of attempts (evolutions). Genetic algorithms, as part of a wider field of artificial intelligence, are being increasingly employed and are indeed successful in dealing with hard-solving issues (NP-hard).
\end{abstract}

Key words: production scheduling, genetic algorithm, genetic algorithm with niches

\section{INTRODUCTION}

Production planning and scheduling (PPS) are founded on production load and capacities over different horizons and on diverse levels by determining flow of materials and use of appropriate resources. PPS are highly interdependent, although they possess their own timescale, resources, activities and specific optimization criteria. Planning provides observance of high-level temporal and resource capacity restrictions and thus assigns goals and also resources and temporal limitations for scheduling. More than that, scheduling is deemed responsible for conversion of production plans into executable agendas in accordance to both detailed resource tasks and operation sequences. Inappropriate scheduling strategy may impede realization of sound plans, while on the other hand, a good scheduling strategy can be executed more appropriately than an insufficient planning strategy (Egri et al., 2004).

Period of production cycle, with reductions in revolving capital and inventory, as well as shipping period, and an increase in equipment usage, may be reduced by reliable production planning and scheduling (Cirovic \& Simeonov, 2010).

Translation of orders issued in manufacturing process into jobs has to be performed in accordance with respective due dates. Commonly, these jobs have to be processed on machines in one a specific order or according to a sequence in a work center. Acknowledging that fact that some machines may be occupied, delays in job-processing may happen. Thus, machine failure or processing times being longer-than-acceptable are only some of unpredicted events on shopfloor. Having great impact on schedules as such, they need to be taken into account (Pinedo, 2005).

Though it is difficult to resolve such complex issues, it is crucial to find efficient support methods and insightful, flexible models that include swift and reliable solution techniques. PPS problems must be treated in an integrated way in spite of superior-inferior hierarchy implemented towards reaching solutions. According to some authors, appropriate solutions should be based on approximate and heuristic algorithms. Methods including tabu search, beam search, ant colony algorithm, simulated annealing algorithm and genetic algorithm are still restricted and aimed solely at solving particular issues. For instance, to find solution in an efficient way, tabu search and beam search utilize a search strategy. In that sense, simulated annealing algorithms prove more efficient in local search, while genetic algorithms score much higher in global search (Zhang \& Yan, 2003). For that matter, some authors favor a mixed strategy as such. For example, Jwo opted for genetic algorithms for global search and made use of simulated annealing algorithms for local search (Jwo et al. 1999).

\section{PRODUCTION SCHEDULING}

Problem of scheduling is often associated with allocation of resources in order to optimize one or more functions of aim.

Production scheduling, in its exact meaning, consists of initiation, observation and respective correctional procedures (so as to certify performance in terms of quantities, dates, quality, costs and work conditions). Production targets emanate from goals of production scheduling. Most frequent aims are, for example, directed towards meeting respective deadlines of shipments, growing usage of capacities, decreasing interphase stocks, reducing cycles of production, etc.

Scheduling problem can be characterized as simple or easily solvable only if there is an algorithm which in polynomial time can produce an optimal solution. However, to create an algorithm which is to solve a specific problem is not sufficient in itself. Thus, finding a solution may require a longer period which is directly dependent on problem size.

As theory of complexity has certified that scheduling problem is part of the group of NP-hard problems, completely different approach is thus necessary in optimization of scheduling.

\section{GENETIC ALGORITHM}

Genetic algorithms (GA), as heuristic method of optimizing inspired by evolution, function through inscribing potential solutions in forms of structure very similar to that of chromosome. Each chromosome is compiled of genes which correspond to characteristics of a given problem. As the problem becomes more complex, number of genes increases thus the related chromosome becomes bigger. Overall numbers of all chromosomes which represent potential solutions of a given problem are termed population.

GA were developed in 1975 by John Holland and colleagues from the University of Michigan. Algorithms based on principals of natural selection and genetics are considered to be part of a group of techniques of search space which are specifically directed because they do not search randomly whole space of a respective solution (guided random search technique). Possibility of locating global extreme in search space in which exist higher numbers of local extremes is one of 
the greatest advantages of GA when compared to classic deterministic methods.

Previous experiences with GA point out to several deficiencies. One of the more important deficits is a possible early convergence towards local extreme. Namely, although GAs can successfully avoid local extremes, it may not be reliably claimed that solution reached by use of GA is a global extreme or, on the other hand, only a solution poised nearby such an extreme. This problem was attempted towards solution through several ways ranging from different modes of selection to increasing possibility of maintaining genetic difference of a respective population. However, it has to be noted that for a considerable number of solutions from real life where number of global extremes is not known this is not a particularly urging matter simply because solutions reached are "good enough." Thus, this method is exceptionally 'stable' and consequently applied, with minimal modifications, to different problems.

Use of GA in solving problems of scheduling appeared ten years after John Holland and his colleagues formulated basics of GA. L. Davis was the first one to attempt solving the problem of allocation by using algorithms. This attempt was readily followed by other researchers. Reason for such an enormous interest is easily assigned to the fact that the problem of allocation is present in many different areas of human existence.

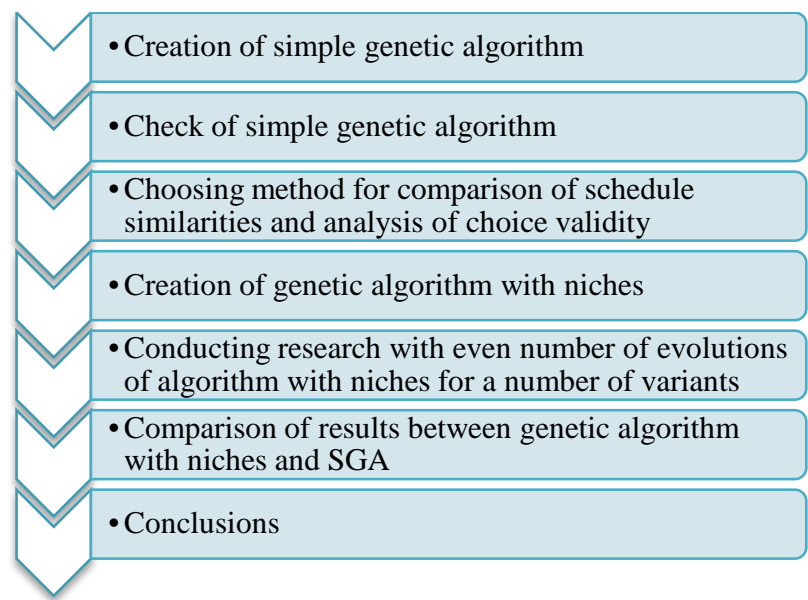

Fig. 1. Mode of comparison of simple genetic algorithm (SGA) and genetic algorithm with niches

\subsection{Genetic algorithms with niches}

Aim of GA with niches is division of population according to greater number of stable groups (niches) inside which it is essential to find appropriate solution. In search space in which there is greater number of local optimums possibility of simultaneous generating and maintenance of higher number of solutions through entire stream of evolution in order to avoid congestion of algorithms in one of the local optimums is indispensable. Finding global optimum compared to local ones is not only characteristic of algorithms with niches. For that matter, each GA which bears less pressure of selection has greater chances of finding global optimum. However, problem with algorithm set in this particular way is that such an algorithm is searching larger space of problem definition, but it cannot be focused on good solutions. On the other hand, increasing pressure of selection results in premature convergence, which, in itself, is also not desirable. Solution to this seemingly unsolvable problem lies in forming niches possessing 'restoration pressure.' Namely, it is possible to enlarge pressure of selection inside niches without premature convergence on a global level to be resulting from it.

Fig. 1. shows method of comparison of SGA to genetic algorithm with niches. Due to comparison of two algorithms, SGA will be initially created and this will be followed by setting optimal parameters of algorithm (population size, number of evolutions, type of crossover operators, and so on). GA will be repeated as many times as it is necessary for most appropriate solutions from each try to be implemented into the Gauss curve. Thus, in this way measurement of dissipation of solutions reached will be revealed. Before creating GA with niches it is necessary to find modes of comparison of two schedules in order to discern their affiliation to a respective niche. In that sense, it is required to generate several different variants of GA with niches so that, after completing research, it would be possible to affirm if specific variant with niches is better than SGA algorithm. Due to comparison with SGA, same number of repetitions of every chosen variant of algorithms with niches will be executed aiming at analysis of dissipation of results.

GA are directed at effectively reducing the holding costs of the work-in-process inventory, the production costs, the penalty costs of the parts which are over-/underproduced, as well as the tardiness time costs and the setup costs, in order to fully take advantage of the earliness time.

\section{CONCLUSION}

Genetic algorithms, as part of a wider field of artificial intelligence, are being utilized more often in solving problems of real life. Greater interest in genetic algorithms is deducted from the fact that genetic algorithms are indeed successful in solving problems that belong to the category of hard-solving issues (NP-hard)

Better knowledge of selection mechanisms and development of new variants of genetic crossover operators so as to deal with permutation problems has introduced genetic algorithms to one of more important segments of optimization techniques. In that respect, due to simplicity and reliability, genetic algorithms possess great potential for improvement and thus deserve more attention and investment of both academic community and industry.

\section{ACKNOWLEDGEMENTS}

This paper was realized with financial support from the research project number FSI-S-11-5.

\section{REFERENCES}

Cirovic, I.; Simeonov, S. (2010). Approaches for Solving Production Planning and Scheduling Problems Using Integrated Heuristic Methods, Proceedings of the 21th International DAAAM Symposium, 4 th International DAAAM Conference, Zadar, ISSN 1726-9679, ISBN 9783-901509-73-5, Katalinic, B. (Ed.), pp. 1151-1152, DAAAM International Vienna, Vienna

Egri, P.; Kovács, A.; Márkus, A. \& Váncza, J. (2004). Projectoriented Approach to Production Planning and Scheduling in Make-to-order Manufacturing, Available from: www.sztaki.hu/ akovacs/publications/psie2005.pdf Accessed: 2010-04-20

Jwo, W. S.; Liu, C. W. \& Liu, C. C. (1999). Large-scale optimal VAR planning by hybrid simulated annealing/genetic algorithm. International Journal of Electrical Power \& Energy Systems, Vol.21, No.1, (January 1999) page numbers (39-44), ISSN 0142-0615

Pinedo, L. M. (2005). Planning and Scheduling in Manufacturing and Services, Springer Science+Business Media, Inc., ISBN 0-387-22198-0, New York

Zhang, X. D. \& Yan, H. S. (2003). Integrated optimization of production planning and scheduling for a kind of job-shop. The International Journal of Advanced Manufacturing Technology, Vol.26, No.7-8, (October 2005) page numbers (876-886), ISSN 0268-3768 